\title{
EL URBANISMO CONTINGENTE EN LAS OBRAS PÚBLICAS Y EL GOBIERNO URBANO Barranquilla en las décadas de 1950 y 1960
}

\author{
Óscar Iván Salazar Arenas \\ Departamento de Sociología \\ Universidad Nacional de Colombia, Sede Bogotá \\ oisalazara@unal.edu.co
}

\section{RESUMEN}

Este artículo elabora una discusión sobre el urbanismo contingente, como parte de las prácticas del gobierno urbano centrado en las vías de las ciudades (calles, avenidas, andenes) y otras obras públicas (alcantarillado y canales de aguas lluvias). Se presenta además como herramienta conceptual para describir y comprender la manera como se produjo el espacio urbano en una de las ciudades colombianas más importantes en el siglo XX. Se utilizan casos concretos de reformas urbanas en Barranquilla, la segunda ciudad más grande del país y el puerto más importante a inicios de la década de 1950. Como conclusión del análisis se señalan cinco características del urbanismo contingente en torno a: su relación con el urbanismo planificado o académico; su relación con los procesos de modernización; su alcance temporal y espacial; la dimensión estética y formal; y el papel de lo técnico y lo político.

Palabras clave: Urbanismo contingente, Barranquilla, historia urbana, infraestructura

\begin{abstract}
This paper makes a discussion about the contingent urbanism, as part of governing urban practices focused on the operation over the roads of the city (streets, avenues, sidewalks, etc.) and other public works (sewage system and rain gutters). It is also presented as a conceptual tool for describing and understanding how the urban space was produced in one of the most important Colombian cities in the $20^{\text {th }}$ Century. The paper focuses on specific cases of urban reforms in Barranquilla, which was the second biggest city of the country and the most important harbor at the beginning of 1950's. As a conclusion of the analysis, 5 characteristics of the contingent urbanism are presented, toward the following aspects: it's relation to the planned or academic urbanism; it's relation to the modernization processes; it's temporal and spatial scope; it's aesthetic and formal dimension; and the role of technics and politics.
\end{abstract}

Key words: Contingent urbanism, Barranquilla, urban history, infrastructure 


\section{INTRODUCCIÓN}

En la historia del urbanismo de las ciudades latinoamericanas del siglo XX se suelen plantear miradas dicotómicas que sitúan en el lado deseable a la construcción planificada de la ciudad y en la indeseable a la informalidad. De otro lado, en los recuentos históricos sobre el urbanismo y sus teorías es posible encontrar algunas descripciones sobre los efectos reales de la implementación de los planes y las obras públicas, pero el énfasis sigue siendo el de resaltar los discursos y actores que impulsan las intervenciones urbanas de grande o mediana escala (Ascher, 2004; Hall, 1996; Mongin, 2006).

En contraste con esas perspectivas, este trabajo propone hacer énfasis en la dimensión de la implementación y los efectos de la planificación como práctica de gobierno de las ciudades. Esto supone cambiar de perspectiva para concentrarse en formas concretas de co-producción de la ciudad en el cruce de diferentes intereses, agentes y prácticas de gobierno. Mi hipótesis en este texto es que buena parte de las maneras de producir el espacio urbano en el siglo XX no se pueden entender adecuadamente desde las dicotomías tradicionales formal / informal, o planeado / no planeado, sino que es necesario ver el proceso de producción como un ensamblaje que hace coexistir lo que se ha visto como separado. Desde esta visión, la ciudad es resultado de diversas líneas de fuerza, intereses contrapuestos, discursos y saberes que resultan más bien "ensamblados" de formas ambivalentes (Amin \& Thrift, 2002; Farias \& Bender, 2010; Graham \& Marvin, 2001; Latour, 2008).

El concepto de "urbanismo contingente" permite comprender una parte de esas formas ambivalentes de ensamblaje, que conjugan la planeación y la improvisación, y la visión de largo plazo con las visiones políticas del corto plazo. Mostraré los vínculos del urbanismo contingente con el concepto más general de "gobierno de las vías", que alude a una forma de gobierno que opera por medio de un conjunto de saberes que actúan sobre las calles y vías de la ciudad, con el objetivo de gobernar a la población por medio de la regulación de las movilidades urbanas (Foucault, 2006; Jensen, 2009; Salazar, 2016; Salter, 2013; Urry, 2007). En gran medida, este ejercicio se relaciona con los estudios sobre infraestructura, y concretamente con aquellos que analizan sus dimensiones política, estética y simbólica (Larkin, 2013). En esta perspectiva, más que los planes y proyectos, se rastrean las prácticas y los efectos de la co-producción sociotécnica de la infraestructura urbana.

Para desarrollar mi argumento presentaré casos de construcción de algunas vías y obras públicas en Barranquilla, Colombia, durante las décadas de 1950 y 1960, periodo en el que las ciudades más grandes del país atravesaron por una de las etapas de modernización material del espacio urbano más intensas del siglo XX (Aprile-Gniset, 1992; Saldarriaga, 2000). Me concentraré especialmente en el caso de la ampliación de la Avenida 20 de Julio y la canalización de algunos de los arroyos, para ilustrar la manera como la ciudad se fue reensamblando de a pedazos, mediante lo que llamo "urbanismo contingente", y no como resultado de un gran plan de reforma urbana. Se trata de un ejercicio de análisis de fuentes documentales, principalmente prensa de la época, en las cuales fue posible rastrear las controversias que se dieron en medio de las obras y algunas de sus consecuencias o efectos.

\section{URBANISMO, PLANIFICACIÓN Y GOBIERNO DE LAS VÍAS}

\subsection{Del proyecto urbano al gobierno de las vías}

Conviene empezar con una aclaración parcial de términos y perspectivas. De acuerdo con Brand (2001), el urbanismo "estudia las formas urbanas y de vida urbana... [y] permite indagar, soñar y proyectar sin compromisos definidos", mientras que la planeación o planificación urbana se refiere a "aquél conjunto de prácticas del Estado local para regular la organización socioespacial de las ciudades, apoyado en la legislación y las instituciones públicas" (Brand, 2001, p. 21). De otro lado, la historia urbana se refiere a una gran cantidad de fenómenos que implican tanto la construcción física de las ciudades, como su configuración política, económica, social y cultural. Este trabajo se sitúa en el complejo cruce de las tres dimensiones 
(urbanismo como proyecto ideal, planificación y producción concreta de la ciudad), y se encuentra estrechamente vinculado a lo que se ha denominado "urbanismo moderno". Por urbanismo moderno se entienden aquí un tipo de ideas e intervenciones físicas que proyectan las ciudades hacia el futuro y que la imaginan o pretenden reformarla como parte de un impulso y un deseo por lo nuevo, por el afán de progreso, por la apuesta funcionalista y por la organización racional del espacio y las dinámicas sociales. Este tipo de urbanismo tiene además orígenes y vínculos estrechos con la industrialización, los cambios socioeconómicos propios del capitalismo, e implica una apuesta por la aceleración de la vida y la movilización de personas, mercancías y objetos. Pero el término "urbanismo" utilizado aquí alude también a las ideas y modelos de ciudad explícitos o subyacentes a las prácticas de la planificación urbana.

Si bien la producción práctica del espacio, de la que suele ocuparse la historia urbana, no es lo mismo que el urbanismo como proyección o imaginación, desde el siglo XIX muchas ciudades han atravesado procesos de reformas urbanas a partir de proyectos que remodelan parcialmente su morfología. Se trataba de un tipo de urbanismo nuevo, que no operaba para crear modelos ideales de ciudad desde cero como ocurrió en el Renacimiento, sino de un urbanismo reformador, orientado por el principio de la "destrucción creadora" del impulso modernizador (Berman, 1988). La planificación urbana que se consolidó en el siglo XX ensambló en sus prácticas la proyección física de la ciudad, la economía, la estadística, la demografía y la sociología, entre otras disciplinas. Pero ante todo, se constituyó en una práctica de gobierno que apostaba por vincular el proyecto ideal con la producción material de la ciudad, y en la mayoría de casos centradas en las reformas de grandes vías (avenidas, bulevares, calles importantes), es decir, un "gobierno de las vías" con efectos en la regulación el orden urbano, los ritmos y el funcionamiento interno de la ciudad. En tal sentido, situados al nivel de las prácticas de gobierno del espacio urbano, la historia de la ciudad se mezcla con ideas y proyectos que provienen del urbanismo, y en realidad resulta muy difícil sostener que existe una separación tajante entre historia urbana e historia del urbanismo en el mundo occidental.

Si bien no son lo mismo, el desarrollo urbano y las ideas o proyectos de ciudad se vinculan de formas complejas y más o menos estrechas, de acuerdo con el ensamblaje particular de fuerzas políticas y económicas, agentes humanos y no humanos, la acción o incapacidad del Estado local, o la influencia de agentes privados, entre otros factores. Explorar los vínculos entre discurso y práctica en los procesos de producción de las ciudades permite comprender la configuración local de la modernidad, y la idea de "urbanismo contingente" opera como herramienta para comprender los vínculos particulares que se dan en casos como el colombiano entre las ciudades proyectadas o imaginadas, y la producción efectiva del espacio urbano. Al hablar de las prácticas de gobierno que produjeron el Estado, la economía y las naciones modernas occidentales, Foucault (2006) ponía en primer plano el problema de la circulación de sujetos, mercancías y capital. La intensificación de la circulación hacía parte de la emergencia de lo que denominaba gubernamentalidad, entendida como "el conjunto de las instituciones, los procedimientos, análisis y reflexiones, los cálculos y las tácticas que permiten ejercer esa forma bien específica, aunque muy compleja, de poder que tiene por blanco principal la población, por forma mayor de saber la economía política y por instrumento técnico esencial los dispositivos de seguridad" (Foucault, 2006, p. 136). Pero, ¿cómo opera en la práctica esta gubernamentalidad?, ¿qué formas toma y qué fuerzas la moldean en las distintas ciudades en las que el proyecto moderno moviliza ideas, agencias y diversas líneas de fuerza?, ¿Qué tan coherente y monolítico es ese proceso, o qué tan inestable y confuso se torna?

En la historia de estos procesos, los grandes casos emblemáticos de reformas urbanas modernas como las de Haussman en París, Mosses en Nueva York o Cerdá en Barcelona, han dejado en segundo plano en la literatura a otro tipo de intervenciones, menos ambiciosas, menos integrales, quizás fracasadas y aparentemente marginales. Muchas intervenciones prácticas o fragmentarias del espacio urbano que han tenido consecuencias en el mediano o largo plazo parecieran estar por fuera tanto de la historia de las ciudades como de la historia del urbanismo, ya que no son ejemplares, suficientemente monumentales, o se salen de los planes urbanos más generales. Quisiera entonces identificar algunas de las características de ese tipo de reformas, a través del recuento y análisis de la manera como se llevaron a cabo algunas de ellas. Esta identificación de sus características nos permitirá describir y comprender mejor la manera como nuestras 
ciudades han llegado a ser lo que son. Este ejercicio es quizás más pertinente para ciudades latinoamericanas que "entran y salen" de la modernidad (García, 1989), que han sido con frecuencia descritas como casos que no logran consolidar un modelo urbano estable o que han hecho "despegues sin madurez" (Almandoz, 2008).

\subsection{Urbanismo y planificación en Colombia}

Desde los años de posguerra en la década de 1940 y durante la de 1950 se dio en Colombia una transición en la forma de pensar las ciudades centrada en el urbanismo desarrollada por arquitectos, a lo que se denominó planificación integral o planeación comprehensiva (Cortés, 2007; Ramírez, 2011, pp. 116-118). En la primera encontramos a los urbanistas modernos y las primeras generaciones de arquitectos de la Universidad Nacional, muy influenciados por el trabajo del arquitecto Karl Brunner y posteriormente por los Congresos Internacionales de Arquitectura Moderna (CIAM). En la etapa de la planeación integral o comprehensiva los factores económicos se volvieron determinantes. La década de los años 1950 fue también un periodo de consolidación de prácticas de planeación basadas en criterios técnicos y el surgimiento de instituciones especializadas como El Departamento Nacional de Planeación (DNP) y el Departamento Nacional de Estadística (DANE), centrados en la medición y producción de estadísticas para el gobierno de la población.

Sin embargo, mientras eso ocurría, el gobierno urbano apelaba aún a viejas prácticas, y buscaba resolver el día a día los problemas derivados de la expansión acelerada y la llegada masiva de migrantes provenientes del campo. Ya fuera por obra de agentes privados, o gracias al impulso de la administración municipal, se llevaron a la práctica varias reformas urbanas que no obedecían directamente a los modelos de planificación, ni seguían un plan general o concepción integral de las ciudades. Es en este cruce en el que el "urbanismo contingente" puede ayudar a describir y comprender las formas que tomaron el gobierno local y el espacio urbano; pero no quisiera comenzar con una definición, sino proceder a una construcción progresiva del concepto a través de los casos. Para empezar, diré que el término tiene la intención expresa de redefinir los juicios sobre la "improvisación" y la "falta de planeación" en muchas de nuestras reformas urbanas. Este tipo de apelativos fueron muy frecuentes en Colombia por parte de arquitectos como Carlos Martínez, quien desde la revista PROA hizo una crítica constante a la manera de gobernar y dar forma a las ciudades, y se han seguido repitiendo en la historiografía del urbanismo colombiano (Cortés, 2007).

Si bien desde un punto de vista puramente técnico, muchas obras como los ensanches de las vías, o el inicio de la construcción de una avenida puedan ser vistos como improvisación, en el mediano y largo plazo esas decisiones tuvieron efectos notorios en la forma urbana y no necesariamente se revirtieron a pesar de sus fallos. Adicionalmente, procuraré sustentar la idea de que a pesar de la "falta de planeación" y la "improvisación" este tipo de urbanismo no carecía de una visión de futuro. Incluso puede observarse una relación estrecha con las ideas de la planificación, ya que muchas de las acciones de los gobiernos locales interpretaron esos planes, aunque el resultado final contradiga los planes. Además, esas obras fueron una forma de urbanismo práctico y de gobierno del espacio cuyos efectos materiales se ensamblaron con la ciudad preexistente y fueron también antecedente para la que le siguió.

\subsection{Desarrollo urbano y modernidad en Barranquilla}

A diferencia de la mayoría de las ciudades importantes en Colombia, Barranquilla no tuvo un origen planificado como parte del urbanismo español de la colonia, sino que surgió como un poblado de viajeros, comerciantes y ganaderos a orillas del río Magdalena en una fecha incierta que algunos autores han situado en el siglo XVII. Durante la segunda mitad del siglo XIX Barranquilla adquirió una importancia nacional de primer orden tras la construcción del muelle marítimo de Puerto Colombia y su conexión por vía férrea a la ciudad que se puso en servicio en 1893 (Nichols, 1973, p. 120). Se constituyó así en el puerto más importante por su 
conexión con el Río Magdalena, que era prácticamente la única vía de ingreso y salida hacia el interior del país.

La ciudad creció con una estructura de damero irregular, inicialmente en torno a lo que se llama la "vía ancha", y que actualmente es el Paseo Bolívar, sin mayor planeación que la adición progresiva de nuevas manzanas a la cuadrícula y el crecimiento predio a predio. A principios del siglo XX Barranquilla fue la primera ciudad del país que construyó barrios planificados. El primero de ellos y el más importante por su tamaño y concepción fue el Barrio El Prado (1922), construido por la Compañía Urbanizadora de El Prado, conformada por empresarios de las élites barranquilleras, como las familias De la Rosa y Parrish. Esta empresa se constituyó en una urbanizadora de carácter privado de gran éxito, que además de El Prado construyó más de 20 barrios de la ciudad hasta la década de 1980 (Vergara \& Vidal, 2009, pp.25-26). Al mismo tiempo, el crecimiento tradicional predio a predio continuó desarrollándose, y durante las décadas de 1950 y 1960 la ciudad vio nacer y expandirse varios barrios informales como parte de la acelerada migración de población rural a la ciudad.

El impulso de urbanización privada iniciado en la década de 1920 no estuvo regulado u orientado por un plan general de la ciudad que se hubiese materializado en un proyecto. A pesar de que desde la década de 1950 la ciudad hizo esfuerzos por hacer un Plan Piloto y creó dependencias dentro del gobierno municipal para regular la construcción de la ciudad, la materialización de esa construcción en lo relativo a la vivienda dependió casi siempre de los agentes privados. Además, para el periodo que cubre este artículo, el cuidado de lo que hoy llamamos espacio público (parques, andenes, plazas, arborización, embellecimiento de fachadas), estuvo a cargo de la Sociedad de Mejoras y Ornato, una entidad privada en la que participaban familias de empresarios y gobernantes de la ciudad. Para el funcionamiento cotidiano de las movilidades y los ritmos urbanos, la ciudad dependía de empresas privadas de buses, taxis, y automóviles particulares, además de quienes se desplazaban a pie o en bicicleta. Por su parte, las vías y los servicios públicos eran el único campo en el cual participaba directamente el gobierno local, por medio de las Empresas de Servicios Públicos, que hicieron levantamientos de mapas y un ejercicio de planeación aplicado a los servicios específicos de su competencia, tales como la pavimentación de vías, acueducto, alcantarillado y canalización de arroyos.

Además de su importancia económica, en la primera mitad del siglo XX, Barranquilla constituía un importante referente de cultura cosmopolita por sus vínculos con el exterior, un buen número de migrantes extranjeros que vivían o pasaban regularmente por la ciudad, y contaba también con un incipiente, pero pionero desarrollo industrial. La idea de modernidad que se materializó en Barranquilla en este periodo estaba marcada, por un lado, de una gran influencia norteamericana, la adopción de estéticas de lo que se ha denominado "movimiento moderno" en arquitectura (Bell-Lemus \& Gamboa, 2003), y la modernización de la infraestructura de la ciudad por medio de las Empresas Públicas, que en las décadas 1930 y 1940 expandió los servicios públicos y la infraestructura (Ospino, 2003, p.27). En este sentido, la modernidad local de Barranquilla se refería tanto al modernismo -la dimensión cultural y estética de los gustos y las prácticas, principalmente norteamericanas-, como a la modernización material y física de la ciudad -que remitía a la confianza en la técnica para mejorar las condiciones de las vías, los servicios públicos y la construcción de nuevas edificaciones (Caballero, 2000).

A lo largo del siglo $X X$, sus periodos de auge o crisis han estado relacionados directamente con el puerto de la ciudad, el río Magdalena y la política económica nacional. Sus mejores años de crecimiento fueron hasta mediados de la década de 1940, mientras fue el puerto más importante del país, y su crecimiento continuó hasta finales de la siguiente década impulsado por el auge industrial y comercial. A partir de la mitad de los años 40 y hasta finales de los 50 la ciudad vivió la etapa final de un crecimiento económico continuo y de un desarrollo urbano y de obras públicas que la mayoría de las ciudades del país no habían tenido hasta ese momento. Comenzando la década de 1950 Barranquilla tenía poco más de 276 mil habitantes, y era la segunda ciudad en tamaño de población después de Bogotá, con alrededor de 638 mil personas. Llegó a tener el segundo PIB más alto de Colombia, precedida únicamente por Bogotá, pero su economía entró en declive especialmente desde 1958 (Bell-Lemus, 2008). Así, los años 50 correspondieron a una tendencia de 
crecimiento en medio de una "prosperidad de milagro", mientras que la década de 1960 se caracterizó por una crisis que se mantuvo hasta mediados de la década de 1970 (Bell-Lemus \& Villalón, 1992, pp. 75-93; Meisel, 1987, pp. 55-84).

\section{URBANISMO CONTINGENTE EN BARRANQUILLA}

\subsection{El plan de Fomento para el Atlántico}

En 1952 se publicó el "Plan de fomento para el Atlántico", el primer documento del recién creado Consejo Nacional de Planificación, que luego se convertiría en el Departamento Nacional de Planeación. La mayoría del plan se ocupaba de Barranquilla, e incluía dos aspectos relacionados con las movilidades y el transporte: las carreteras y la pavimentación urbana. De una parte, hacía un diagnóstico de la situación de desconexión terrestre de la ciudad respecto a las regiones circundantes y frente al resto del país, y presentaba un plan para la construcción de carreteras departamentales, que en ese momento eran prácticamente inexistentes. El informe señalaba que estos factores estaban incidiendo en la desaceleración de su economía (Currie \& Peñalosa, 1952, pp. 9-10).

Uno de los temas que resaltaba el plan de fomento era el de la pavimentación de las calles de la ciudad. Aunque se había avanzado bastante, todavía más de la mitad se encontraban destapadas. El Plan de fomento decía al respecto: "La pavimentación es más urgente en Barranquilla que en la mayoría de las grandes capitales del país, debido a las lluvias extraordinariamente fuertes durante el invierno. Ellas causan fuerte erosión y depositan grandes cantidades de arena en las calles centrales. De los 500 kilómetros de la ciudad, sólo 200 están pavimentados" (Currie \& Peñalosa, 1952, pp. 21-22).

La pavimentación servía a varios propósitos, incluido el alcantarillado pluvial, debido a la dificultad para implementar un sistema subterráneo en la ciudad. Debido a que la ciudad está situada a orillas de la desembocadura del río Magdalena, en una meseta inclinada, durante las temporadas de lluvia las calles se inundaban y se convertían en arroyos que desembocaban en los caños que conducían al río. Estos arroyos representaban un gran peligro para peatones y automovilistas, además de dificultar el paso por el flujo de agua, barro y otro material arrastrado por la corriente. Sin embargo, a pesar del impacto del invierno, "según las Empresas Públicas, la paralización del tránsito por causa de las lluvias no alcanza[ba] a más de un total de 24 horas por año" (1952, p. 23). El plan de fomento contemplaba pavimentar 90 kilómetros de vías en cinco años, la mitad en asfalto y la mitad en cemento, financiados por medio del impuesto de valorización. El informe decía que la pavimentación se había hecho siguiendo el curso de los arroyos para que sirvieran como canales de aguas lluvias y proponía diversificar el programa. Formulaba la necesidad de continuar con la pavimentación en las calles por las que corrían los arroyos, y construir además canales que los condujeran durante el invierno en sentido oriente occidente para desembocar en el río y evitar que las aguas lluvias corrieran por el centro de la ciudad (1952, 23-24). 


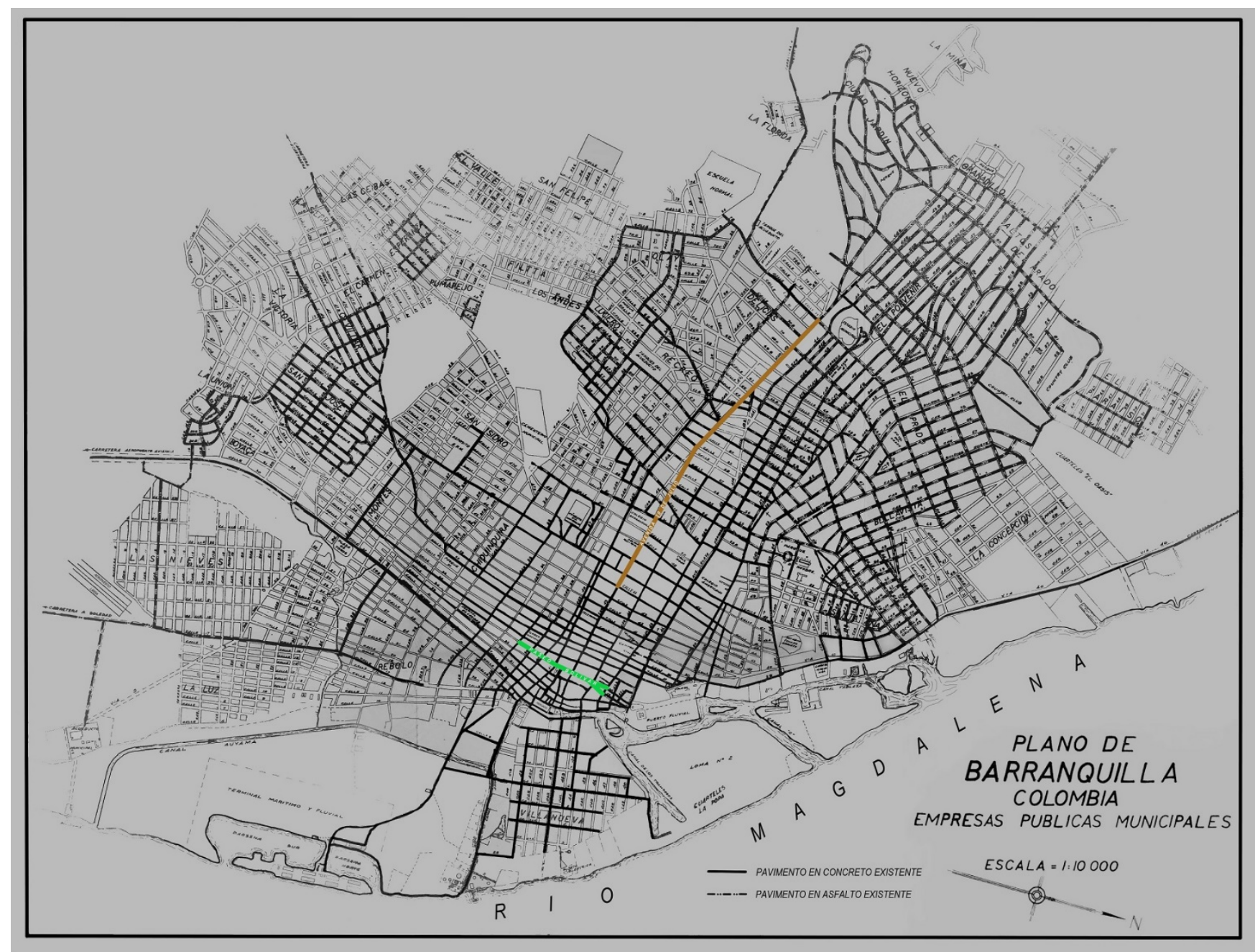

Plano de Barranquilla con el Paseo Bolívar y Av 20 de Julio resaltadas

En verde se muestra la calle 34 y el Paseo Bolívar, y en naranja el proyecto de ampliación de la Avenida 20 de Julio adelantado entre 1955 y 1959 (elaboración propia sobre plano de las calles pavimentadas en Currie \& Peñalosa, 1952).

\subsection{Las ampliaciones de la Avenida 20 de julio}

Además de los esfuerzos constantes por avanzar en la pavimentación de las calles, la ampliación de algunas de las vías del centro se había vuelto una necesidad, especialmente del Paseo Bolívar y su zona circundante. La pavimentación se hacía por tramos cortos y con presupuesto limitado, que en algunos casos era completado por empresas privadas interesadas en la pavimentación, como en el caso de la Vía 40 ("305 mil pesos" 1958, agosto 2). Sin embargo, la ampliación de la Avenida 20 de Julio constituyó uno de los casos de renovación urbana y modernización de vías más importante del centro en la década de 1950, no solo por su importancia para acceder al centro y al Paseo Bolívar, sino porque en ella se aplicó por primera vez el modelo del impuesto de valorización para financiar las obras. La ampliación había sido ordenada por la Oficina de Valorización del municipio de Barranquilla en 1949, junto con la creación del respectivo impuesto. En 1951 se ordenó su cobro ("Dos millones 171 mil pesos" 1951, enero 16), y en 1955 se dio inicio oficial a las obras, que cubrieron el tramo entre las calles 44 y 72 (“La ampliación de 20 de Julio" 1955, mayo 11).

Al finalizar la obra en 1959, habían ocurrido una serie de cambios evidentes respecto al plan original: el trazado de la vía se había modificado varias veces, las demoliciones y compras de predios habían tomado mucho más de lo presupuestado, los costos se elevaron, y el resultado fue una calle con distintas especificaciones técnicas en varios de sus tramos, incluyendo anchos de calzada, alturas de los andenes y diferente tratamiento respecto a las fachadas de los edificios (Gerlein Comelín \& Gómez Támara, 1958, julio 23). A pocos días de la apertura de uno de los tramos cercanos al Paseo Bolívar la prensa hablaba de 
favoritismos para algunos propietarios a quienes se les había permitido mantener sus edificaciones, los balcones o fachadas originales, mientras que a otros se los había obligado a demoler ("La ampliación de 20 de Julio," 1959, diciembre 2). En 1959 Juan Goenaga señalaba cómo la calle en uno de sus extremos tenía un ancho tal que cabían cuatro automóviles y progresivamente se iba cerrando para terminar en un cuello de botella por el cual escasamente cabía sólo uno y se prestaba para gran cantidad de accidentes. La ampliación había expandido la calle de 6 a 19,50 metros en su sector más ancho, pero con una forma irregular y tramos muy angostos (Goenaga, 1959, diciembre 24).

Veamos un poco más en detalle el asunto del ancho de la vía. Una de las controversias más fuertes de la obra fue el tema del ancho de los andenes y el ancho de la calzada para los automóviles. Los propietarios de los negocios y predios contiguos a la obra argumentaban que debían construirse calzadas anchas para evitar los trancones y dar vía libre a los vehículos. Sin embargo, dentro de los planes se proyectaba construir andenes de 3,75 metros de ancho como norma para toda la ampliación de la vía en ambos costados, lo que obligaba a reducir el ancho de la calzada. Según dos ciudadanos afectados por las obras, "los estudios técnicos, las bases del urbanismo moderno, constituyeron argumentos oficiales para negar de plano la petición de los propietarios contribuyentes y haber llegado al extremo de haber rechazado la asesoría técnica que se pedía de la Empresas Públicas Municipales". Su descripción del resultado final de las obras muestra las modificaciones al plan: “...los 3 metros, 75 centímetros, que necesariamente, sin posible modificación, imponía la técnica, resultaron un mito. Existe allí un admirable maramagnum (sic) de medidas, desde los dos metros, dos metros con 55 centímetros, tres metros con 12 centímetros, ..., como si se tratara de un laboratorio de experimento para las próximas ampliaciones" (Gerlein Comelín \& Gómez Támara, 1958, julio 23).

En función de mi argumento, quiero resaltar dos características de lo ocurrido con esta avenida. Primero, la obra estaba acorde con las recomendaciones del plan de fomento, que a su vez retomaba y reconocía también el trabajo ya iniciado de pavimentación de las vías. En tal sentido, la concepción de la obra no era improvisada, aunque sí lo fueran varias de las decisiones que se tomaron durante el proceso de construcción. Estas decisiones obedecían a las imprevisiones propias de la construcción de la obra, sobre todo en lo relativo a la compra de predios y las demoliciones. Segundo, el "argumento técnico" se volvió un recurso retórico en la controversia entre las autoridades que ejecutaron las obras y la ciudadanía. Aparece como justificación para las decisiones que se tomaron de parte de las autoridades frente a los propietarios y ciudadanos afectados por las obras, pero ellos también utilizaban la metáfora científica como recurso para argumentar en contra de las decisiones técnicas (recordemos la idea del "laboratorio de experimento"). De forma paralela, el argumento técnico era cuestionado por la evidencia de múltiples decisiones "no técnicas" que beneficiaban a unos y afectaban a otros, sin una aparente explicación distinta a los favoritismos y la influencia de particulares.

El caso de la 20 de Julio en Barranquilla ilustra también las dificultades en la implementación del sistema de valorización en una ciudad que no tenía tradición de planificación urbana. Aunque el sistema de renovación por valorización ya había sido aplicado en el caso de Bogotá en la ampliación de la Carrera Décima, en Barranquilla el resultado final fue accidentado y más complejo, aunque se trataba de una obra de impacto mucho menor. A las ampliaciones de 20 de Julio le siguieron las de la avenida La Paz, la Vía 40 y la calle 72 , entre otras, con el modelo del impuesto de valorización, con dificultades similares y lentitud en el avance de las obras. 


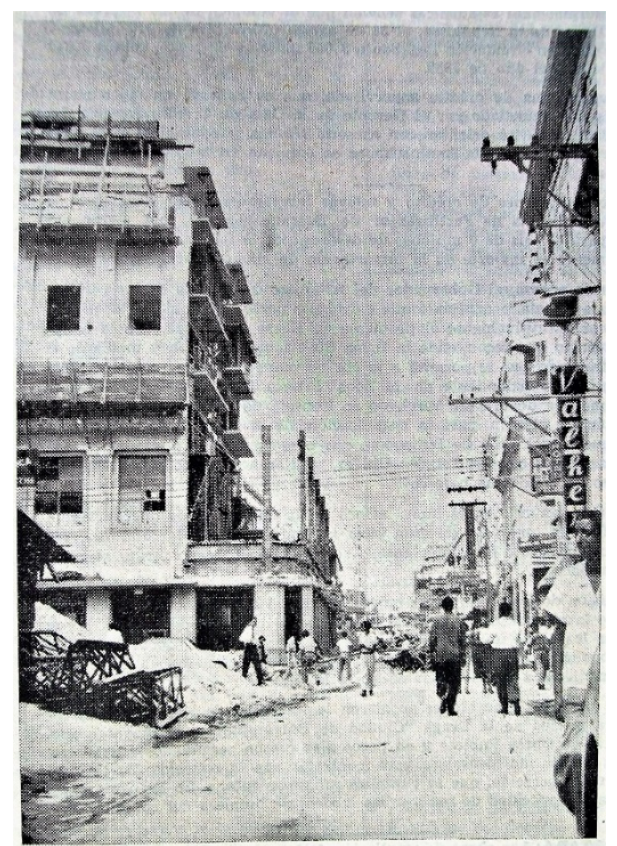

Obras de ampliación de la Avenida 20 de Julio

("Barranquilla progresa" 1959, septiembre 9)

\subsection{Los arroyos y el ensamblaje urbano}

Además de las ampliaciones de vías, otros dos temas críticos de la administración municipal fueron siempre la pavimentación y el mantenimiento de las calles. Durante la década de 1950 hubo al menos dos dependencias que se ocuparon de esta labor, con resultados diferentes. De una parte, las Empresas Públicas estaban realizando pavimentación en concreto, y de otro lado, la Secretaría de Obras Públicas hacía pavimentación en asfalto. El concreto demostró ser más duradero y más resistente a la acción de los arroyos, aunque el asfalto era más rápido de instalar y reparar. El concreto era más costoso, lo cual dividía las opiniones respecto a la conveniencia de uno u otro material, sobre todo cuando las dificultades económicas para la ciudad comenzaron a hacerse evidentes. En 1960 el alcalde Raimundo Sojo decidió introducir una reforma para eliminar la duplicación de esfuerzos, reducir la burocracia y optimizar la realización de las obras. Propuso entonces la eliminación de la Secretaría de Obras Públicas y ordenar la pavimentación únicamente en concreto de las calles por las que corrían arroyos ("Tecnificación de los impuestos" 1960, agosto 9). Sojo había recibido el apoyo público a sus iniciativas por parte del arquitecto Ricardo González Ripoll, quien en ese momento era decano de la Facultad de Arquitectura de la Universidad del Atlántico (Bell, 2003; McCausland, 2010). El decano aseguró que era “...tal vez la primera ocasión que un alcalde se enfrenta[ba] decididamente a los problemas de la ciudad, desde un punto de vista estrictamente técnico" (González Ripoll, 1960, agosto 23).

El arquitecto González Ripoll fue posteriormente alcalde de la ciudad en 1962, cuando continuó el plan de pavimentación en asfalto y experimentó con soluciones parciales al problema de los arroyos, como en el caso del puente pluvial elevado en una de las intersecciones del temible arroyo Felicidad. Recordemos que el Plan de Fomento del Atlántico planteaba alternativas para los arroyos, pero éstas eran de difícil implementación. El puente del arquitecto consistía en una elevación en concreto que permitía el paso de dos vehículos y tenía un estrecho paso peatonal que posibilitaba el cruce del arroyo durante los inviernos. La obra generó polémica porque según sus detractores, era un puente feo y mal terminado que no hacía justicia a la calidad arquitectónica de Barranquilla, mientras que sus defensores resaltaban su carácter funcional y la utilidad que implicaba (Goenaga, 1962, agosto 24). En esta controversia de nuevo emergieron los argumentos técnicos, aunados ahora a la figura de un gobernante-técnico, un arquitecto. Pero ahora el debate se planteaba en términos del sacrificio de la estética a favor de una mirada funcionalista y pragmática. 


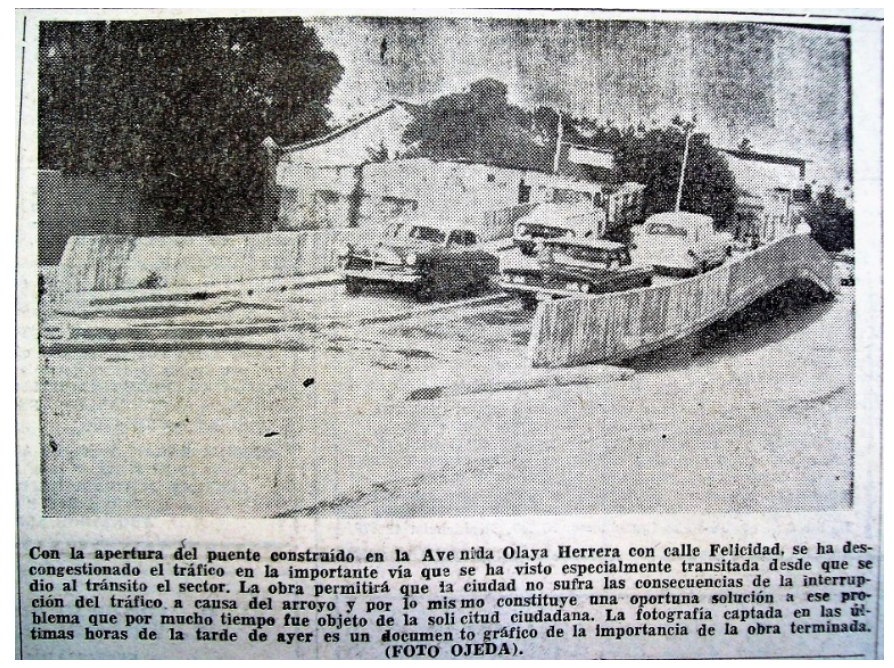

Puente del Arroyo de Felicidad construido por González Ripoll

("El puente de Olaya-Felicidad" 1962, julio 26)

Conviene detenernos un poco más en el caso de los arroyos para mostrar la complejidad de las decisiones, actores y prácticas que se ensamblaron en la producción de la ciudad, dentro de las cuales se encontraban las prácticas del urbanismo contingente. Antes de la pavimentación de las calles y la expansión urbana del siglo XX los arroyos de Barranquilla corrían por sus cauces naturales de tierra y arena, donde se filtraba buena parte del agua al subsuelo, previo a su desembocadura en los caños paralelos al río Magdalena ("El 'general' invierno" 1955, octubre 29). Los cauces de los arroyos permanecían secos buena parte del año, excepto en las dos temporadas regulares de lluvias, entre abril y mayo y entre octubre y noviembre. Con el crecimiento de la ciudad, muchos de los arroyos perdieron sus cauces naturales y comenzaron a atravesar la ciudad por las nuevas calles, inicialmente sin pavimentar. El proceso de pavimentación fue intenso durante la década de 1950, a pesar de haberse realizado de manera fragmentada y accidentada, a la manera propia del urbanismo contingente.

La pavimentación tuvo varias consecuencias no planeadas: el caudal de muchos arroyos se agudizó y ganó en velocidad en su nuevo lecho de cemento; intentar cruzar las calles con arroyos o conducir en ellas durante un aguacero fuerte se volvió más peligroso que antes; varios arroyos comenzaron a desbordarse y a producir inundaciones en las partes bajas de la cuenca debido a la velocidad del caudal, lo que generó emergencias frecuentes para los moradores de esos sectores. Casi todos los años se registraban muertos por ahogamiento, familias sin techo y vehículos destruidos. Los arroyos eran descritos en términos de movilidad: cada invierno se hablaba reiteradamente del "bloqueo de la ciudad" por varias horas. Lo que se bloqueaba, evidentemente, era el tráfico; durante el aguacero no era posible cruzar las calles con seguridad, e implicaba un riesgo incluso para los vehículos más grandes como los autobuses, que en numerosas ocasiones eran arrastrados por los arroyos.

\subsection{El Arroyo de Rebolo}

No se puede afirmar que los problemas de los arroyos se originaron con la pavimentación de las calles. Los arroyos ya existían y la pavimentación sumó nuevos actores, humanos y no-humanos, a los problemas que los Barranquilleros veían en ellos. A través del seguimiento de lo ocurrido con el arroyo de Rebolo es posible observar la suma progresiva de actores y la redefinición de los problemas, aunque en apariencia sean los mismos, como decía constantemente la prensa. El caso también muestra la forma contingente, temporal o más permanente de los ensamblados urbanos de las movilidades y sus relaciones con otros aspectos de la vida urbana. El caso de Rebolo es emblemático debido a su tamaño, la importancia de las vías que cruzaba y las acciones y omisiones del gobierno de la ciudad. 


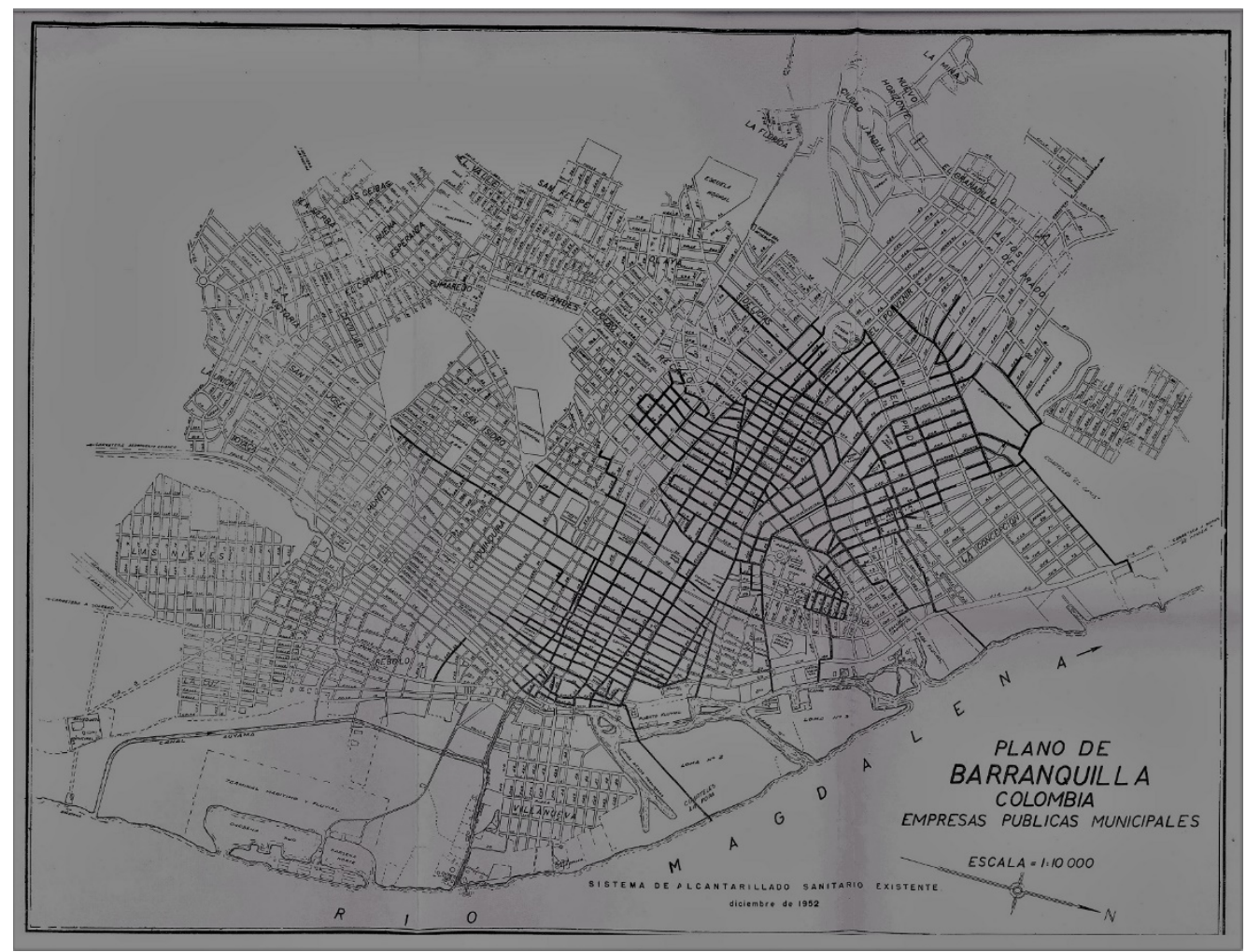

Sistema de alcantarillado de Barranquilla en 1952

Las líneas más oscuras sobre las vías indican la red del sistema de alcantarillado existente (Currie \& Peñalosa, 1952). 


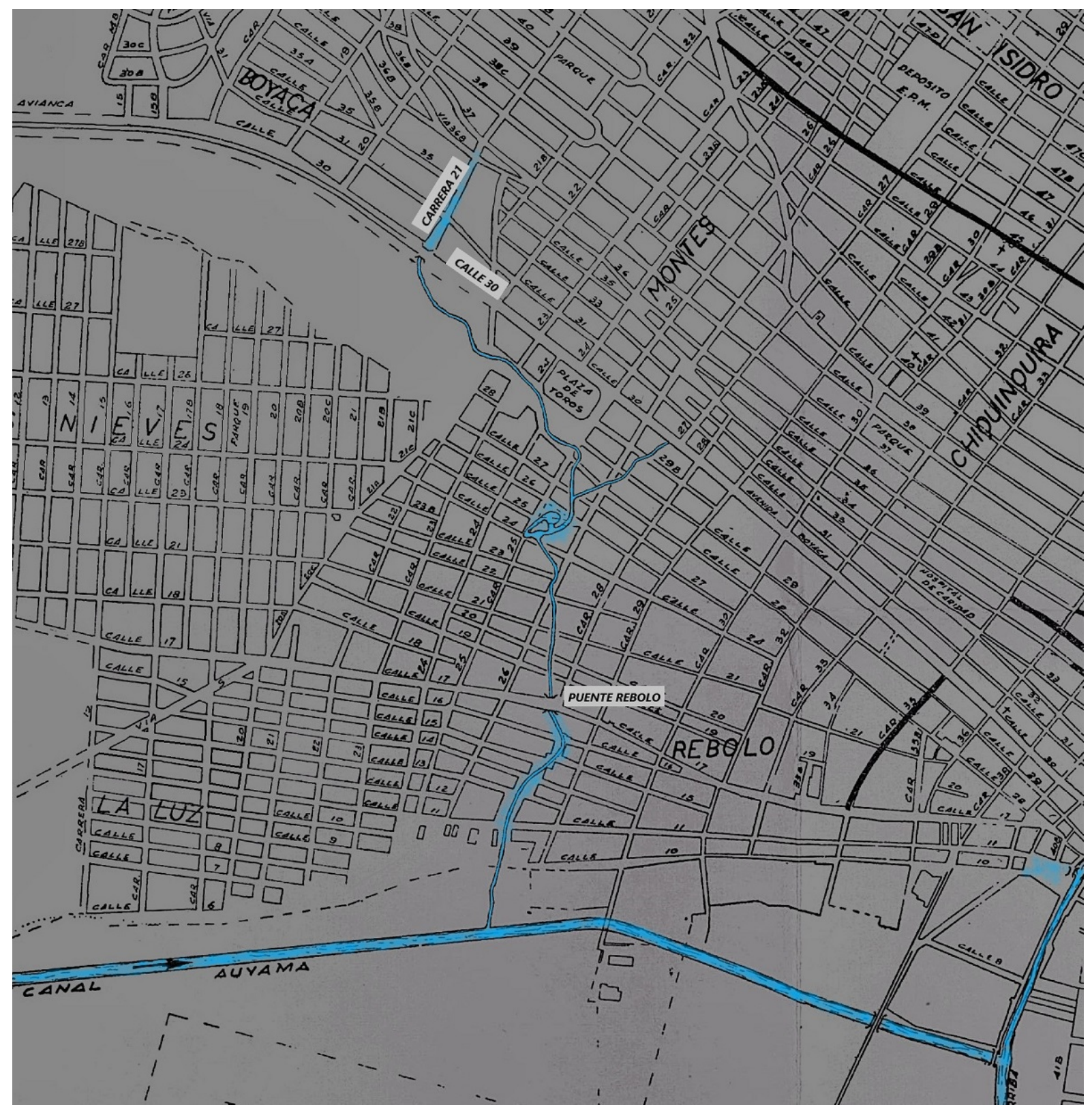

Arroyo de Rebolo a inicios de la década de 1950

En azul se muestra el cauce del Arroyo de Rebolo aún sin canalizar en 1952, que nacía a lo largo de la carrera 21 y desembocaba en al Caño de la Auyama. Algunas de las zonas inundables se muestran en azul por fuera de los contornos del cauce (elaboración propia sobre plano del alcantarillado de Barranquilla en Currie \& Peñalosa, 1952).

El arroyo de Rebolo nacía a lo largo de la Carrera 21 en dirección occidente-oriente, y al pasar entre las calles 30 y 17 atravesaba el sector de la ciudad donde se encontraba el barrio del mismo nombre. El arroyo cruzaba formando más o menos una diagonal hasta la 17, donde se encontraba un viejo y estrecho puente conocido como Puente Rebolo, que era uno de los pocos cruces instalados comenzando la década de 1950. Desde ese punto el arroyo continuaba por la Carrera 27 hasta el caño de la Ahuyama, donde finalmente desembocaba. En 1950 una de las razones fundamentales que motivaban la pavimentación de calles y la canalización de los arroyos era la necesidad de mejorar las condiciones de higiene de la ciudad y el combate a la malaria. La cimentación y nivelación del suelo de los arroyos permitiría eliminar los charcos y pozos de aguas estancadas que durante las temporadas secas se volvían focos infecciosos por la propagación de mosquitos ("Canalización del arroyo" 1950, mayo 10). 
En 1959 las mejoras en pavimentación estaban avanzadas en el sector: existía ya un nuevo puente sobre la calle 30 que conectaba con la vía al aeropuerto, y en la parte más alta del cauce se había hecho una canalización subterránea que en ese punto desembocaba en el lecho tradicional al aire libre que corría hacia el oriente, aún sin canalizar. Las nuevas urbanizaciones al occidente de la calle 30 habían además desviado las aguas lluvias de las calles aledañas hacia Rebolo, lo que aumentó notablemente su cauce en la época de invierno. Dado que no existía alcantarillado fluvial, las calles eran la única salida del agua, y además ahora el arroyo Rebolo estaba sobrecargado recibiendo flujos de agua que antes corrían por otros arroyos y otras calles.

Durante los años sesenta el tramo de la Calle 30 a la desembocadura fue objeto de múltiples planes y proyectos aprobados y rechazados, de obras parciales y contratos adjudicados que después se cancelaban debido a los costos de la obra. La Oficina del Plan Regulador diseñó en 1961 un proyecto de canalización, pavimentación y embellecimiento del arroyo, que incluía la arborización y dotación de mobiliario urbano ("Este es el proyecto" 1961, febrero 25) (ver ilustración). Pero muchas de las obras parciales parecían empeorar el problema. De este modo, en 1962 se hablaba del "monstruo hidráulico" que habían producido la canalización y la sobrecarga de aguas lluvias en Rebolo ("Crecimiento monstruoso" 1962, mayo 17). A pesar de los esfuerzos y de obras temporales para mitigar los daños, el sector entre la 30 y la 17 siguió cobrando víctimas y era lugar de accidentes frecuentes en los días de lluvia cuando vehículos o personas intentaban cruzarlo y eran arrastrados por la intensidad de la corriente.

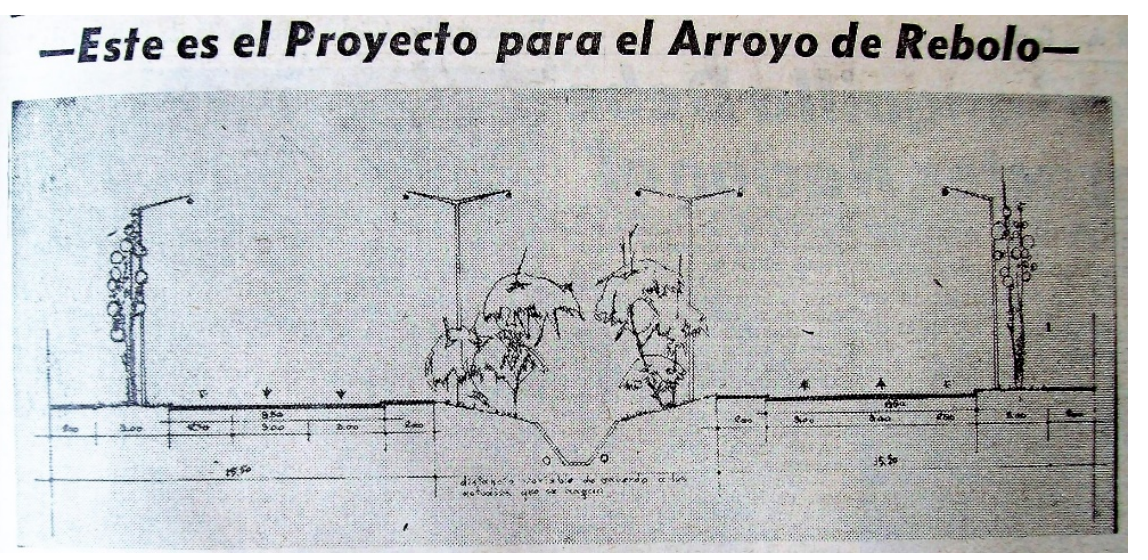

Vista en corte de proyecto para canalizar el Arroyo de Rebolo

("Este es el proyecto" 1961, febrero 25)

El caso de Rebolo muestra cómo el ciclo de coproducción y ensamblaje del espacio urbano genera nuevas dificultades y cómo los sistemas de movilidades pueden incidir o ser afectados por otros elementos y agentes. Los canales y las calles pavimentadas tuvieron efectos en la velocidad y fuerza de la corriente, lo que a su vez impulsaba la construcción de más y mejores puentes en los puntos críticos en que los arroyos se cruzaban con una vía para asegurar que los cruces no se vieran afectados durante las lluvias. El urbanismo contingente hacía parte integral de esta coproducción espacio-temporal de la ciudad, junto con la presencia intermitente de los arroyos; una presencia que era fantasmagórica en épocas de sequía: un espacio sucio y sin construcciones que amenazaba en convertirse por algunos días del año en un río caudaloso.

Desde la perspectiva panorámica del urbanismo planificado, que en el caso de nuestras ciudades solía llegar después de los problemas, lo que debió hacerse fue un plan integral, mucho más general y ambicioso que resolviera y previera las posibles consecuencias y dificultades de las canalizaciones, las urbanizaciones, y la pavimentación de las calles. En este punto surge otra característica del urbanismo contingente. Si bien está vinculado a una visión general de futuro, no tiene la capacidad predictiva o previsiva de la que suele investirse el urbanismo planificado, aunque su visión de futuro sea fantasiosa. Adicionalmente, mientras que el urbanismo planificado es panorámico y busca abstraer grandes secciones o la totalidad del territorio de la ciudad, el urbanismo contingente opera al nivel de la calle, de forma práctica y a corto plazo. 
Las consecuencias de adelantar una obra pública con urbanismo planificado o contingente, como estamos viendo, pueden ser indeseadas y más que resolver para siempre los problemas que buscan resolver, generan nuevas condiciones, nuevas necesidades o problemas antes inexistentes, que a su vez vuelven a convertirse en objeto de controversia y de acción por parte del gobierno de las vías. Para decirlo de otra forma, la intervención sobre el espacio urbano es algo constante y tiende a complejizarse sin que se resuelvan del todo y para siempre los problemas que motivaron las reformas. Por supuesto, es posible juzgar que hay unas mejores soluciones que otras, de acuerdo con lo que queramos evaluar, pero eso no implica que la forma urbana sea eterna. Sólo se estabiliza por tiempos largos o cortos para volver siempre a ser inestable, volverse vieja y obsoleta, o convertirse en un nuevo "estorbo del pasado" que una nueva visión de modernización material tomará como su objeto de operación.

\section{Conclusiones}

Los eventos de Barranquilla que acabamos de revisar no fueron únicos de esa ciudad. En Bogotá, la ciudad del país con mayores cambios físicos y demográficos a mediados del siglo pasado, podrían rastrearse numerosas prácticas del urbanismo contingente. Algunos casos de ellos en el mismo periodo incluyen varias de las decisiones tomadas durante la megaobra de ampliación de la carrera Décima entre 1945 y 1960 (Niño \& Reina, 2010); la pavimentación de la Carrera Séptima luego de los destrozos del Bogotazo el 9 de abril de 1948 (Suárez, 2006); la construcción de la Avenida El Dorado, la Autopista Norte y la Autopista sur, por fuera de las recomendaciones del Plan Piloto y el Plan Regulador (Del Castillo et al., 2008); o los famosos "huecos de Mazuera", que fueron el inicio de los puentes de la Calle 26 (Mazuera, 1972). A pesar de la documentación existente para varias de estas vías bogotanas, las interpretaciones han sido construidas frecuentemente desde la visión panorámica del urbanismo, que dificulta evaluar los resultados de las obras realizadas, así como comprender el proceso de co-producción social y técnica de la infraestructura, ya que nos impone una sola perspectiva. En contraste, el concepto de urbanismo contingente permitiría describir mejor los efectos de las relaciones entre las prácticas de gobierno, los discursos de la planificación urbana, las ideas de ciudad que se intentan materializar y la infraestructura para la movilidad, incorporando las visiones y prácticas de la mayor cantidad de actores posibles. A través de la descripción y análisis de esta forma de producción urbana se puede entender mejor la co-producción efectiva de las ciudades que realizan gobernantes, sistemas de movilidades, sistemas técnicos, grupos de interés y personas en sus prácticas cotidianas. Sinteticemos entonces algunas de las características del urbanismo contingente que se derivan de los casos revisados aquí.

Primero, el urbanismo contingente no es completamente ajeno a la mirada panorámica de la planificación y no necesariamente es su opuesto. En contravía de los ideales del urbanismo académico, muchas intervenciones en calles, avenidas, espacio público y sistemas de transporte que efectivamente se llevaron a cabo resultaron ser interpretaciones -convenientes o no- de la mirada panorámica y de postulados del urbanismo moderno que, sin embargo, no se ajustaban a los planes o incluso los desafiaban abiertamente. Ejemplos de ello son las avenidas modernas de Bogotá construidas en los años 50 por fuera las recomendaciones técnicas de los planes, pero aplicando de manera conveniente todo el conocimiento técnico del uso del concreto y el diseño de las vías. En el caso de Barranquilla, la implementación del impuesto de valorización proponía una novedosa solución técnica al problema de la financiación para las obras públicas, pero su construcción fue moldeada por múltiples intereses y uno de sus resultados fueron calles y andenes desiguales, con especificaciones técnicas diversas.

Segundo, hace parte de un deseo por insertar a las ciudades en el mundo moderno, que interpreta y materializa la modernidad en la infraestructura y el espacio urbano. Es una forma de hacer obras públicas que se inspira, evoca o argumenta su necesidad basada en el deseo de modernizar la ciudad, y en ello se relaciona estrechamente con el espíritu del urbanismo académico. Pero paradójicamente, en un periodo en que en Colombia empezaban a imponerse las ideas del urbanismo de los CIAM con su visión de la ciudad-máquina, de la integralidad y la totalización, la realidad urbana impuso resistencias que nadie planeó. El devenir de los 
gobiernos locales, la expansión acelerada de las ciudades, hacían de nuestras ciudades escenarios muy difíciles de intervenir, en donde se consolidaron tendencias de construcción como la autoconstrucción de vivienda por parte de la población pobre y el urbanismo contingente en las obras públicas, que no se romperían en las décadas siguientes. Estas tendencias no eran ajenas al proceso modernizador, aunque no obedecieran a los planes.

Tercero, opera en la pequeña escala y a corto plazo. Es fragmentado espacial y temporalmente. Esta característica es la que le da su nombre: opera con y sobre las contingencias, generando acontecimientos urbanos que, sin embargo, suelen tener consecuencias de largo y mediano plazo. La pavimentación de las calles y la canalización de los arroyos en Barranquilla son ejemplos claros de esta consecuencia. Las decisiones de las décadas de 1940 a la de 1960 respecto a los arroyos moldearon buena parte de la ciudad que hoy existe. El urbanismo contingente puede operar de forma puntual y no como parte de un plan más general, o una concepción integral del territorio, aunque también tiene lugar en la implementación de planes y proyectos integrales. Es cortoplacista y efectista en sus prácticas, pero incorpora visiones del futuro e ideas sobre lo moderno que se proyectan al futuro y tienen efectos en el mediano y largo plazo. Es "contingente" porque se justifica como solución a problemas prácticos, porque obedece a decisiones, momentos políticos y actores particulares que se encuentran en el proceso real material de la producción del espacio urbano.

Cuarto, el urbanismo contingente incorpora una poética y una estética de la modernización material. Las calles asfaltadas o pavimentadas nos dan el mensaje de que "somos modernos porque la forma de nuestras calles lo demuestra". Se trata también de un bricolaje urbano. La ciudad está armada de pedazos y objetos que provienen de diferentes estéticas, tecnologías y épocas. Además, se trata de intervenciones que en su gran mayoría remodelan o crean espacios e infraestructura para las movilidades urbanas, es decir, las calles, avenidas, canales de los ríos. En tal sentido, no hay nada más "moderno" que remodelar las formas de la movilidad de una ciudad. En el periodo de los años 1950 y 1960 la apuesta decidida fue por impulsar las automovilidades, lo cual implicaba el uso de cemento y asfalto, por encima del hierro, que habría sido el material privilegiado de la modernidad del siglo XIX.

Quinto y último, el urbanismo contingente muestra las estrechas relaciones entre "lo técnico" y "lo político" en la implementación de obras públicas impulsadas por el gobierno local, y cuestiona constantemente las fronteras que se procura imponer entre ambas dimensiones. Es "antitécnico", aunque se basa en el diagnóstico técnico de la necesidad de las intervenciones urbanas; con frecuencia lo ponen a andar gobernantes que sí son técnicos, o que dicen basarse en decisiones técnicas, pero de maneras que no escapan a la controversia técnica. Lo técnico es también un recurso retórico en la contienda política, y es utilizado con diferentes intenciones. En sí mismo el urbanismo contingente no haría parte de una ideología particular, aunque sí de un proceso más general que erige el principio de circulación como base fundamental para la producción del espacio urbano, y en ese sentido es tanto técnico como político.

Lo que algunos autores han denominado "modernización" de las ciudades no fue algo que se hubiera dado de manera uniforme y progresiva. Las tendencias en las prácticas del urbanismo y las prácticas de gobierno no necesariamente corresponden con lo planificado, sino que obedecen a fuerzas en constante juego. En el periodo de las décadas de 1950 y 1960 presenciamos en el país grandes cambios en las teorías y prácticas de planificación, pero también inercias de tradiciones de gobierno e intervención urbana que poco han sido analizadas, o han sido vistas más como las tendencias contra las que habría que imponer formas más racionales y técnicas de producción urbana. Del lado de las tendencias que moldearon buena parte de las ciudades del siglo XX en Colombia, hay al menos tres formas de producción urbana: las obras públicas construidas por fuera de los planes, los cambios e improvisaciones de las obras sobre la marcha, y la construcción privada e informal de barrios, vías y viviendas. Las dos primeras formas de intervenir la ciudad hacen parte del urbanismo contingente. Sin duda, el urbanismo contingente ha tenido un impacto más decisivo en el largo plazo que muchos de los planes urbanos sobre los que tanto se ha escrito en el caso colombiano. El urbanismo contingente permite agrupar y describir más apropiadamente que el urbanismo planificado o académico, muchas de las obras que efectivamente se construyeron y particularmente las vías urbanas. 
Además, hace posible analizar las prácticas del gobierno local y sus consecuencias, en la medida en que nos sitúa en la mediana y la pequeña escala, con el propósito de ver su funcionamiento como instrumentos para el gobierno de la población.

\section{AGRADECIMIENTOS}

Agradezco los comentarios realizados al texto inicial de este artículo por parte de los evaluadores del $\mathrm{X}$ Seminario Internacional de Urbanismo, así como a mis colegas del grupo de investigación Prácticas Culturales, Imaginarios y Representaciones de la Universidad Nacional de Colombia.

\section{BIBLIOGRAFÍA}

305 mil pesos se financiarán para las obras de pavimentación. (1958, agosto 2). El Heraldo, pp. 1-5.

Almandoz, A. (2008). Despegues sin madurez. Urbanizacion, industrializacion y desarrollo en la Latinoamerica del siglo XX. EURE, XXXIV(102), 61-76.

Amin, A., \& Thrift, N. (2002). Cities. Reimagining the Urban. London \& Malden: Polity Press.

Aprile-Gniset, J. (1992). La ciudad colombiana siglo XIX y siglo XX. Bogotá: Biblioteca Banco Popular.

Ascher, F. (2004). Los nuevos principios del urbanismo. Madrid: Alianza Editorial.

Barranquilla progresa. Obras de ampliación de la avenida 20 de julio. (1959, septiembre 9). Boletín de La Cámara de Comercio de Barranquilla, V(173), p. 1.

Bell-lemus, C. (2008). Industria, puerto, ciudad (1870-1964). Configuración de Barranquilla. Apuntes. Revista de Estudios Sobre Patrimonio Cultural, 21(1), 62-73.

Bell-Lemus, C., \& Villalón, J. (1992). Historia de Barranquilla. El periodo del Frente Nacional y la crisis de los años sesenta (1957/58-1974/75). Ensayos de Economía, 3(1), pp. 75-93.

Bell-Lemus, C., \& Gamboa, P. (2003). Arquitectura: El movimiento moderno en Barranquilla 1946-1964. Barranquilla: Universidad del Atlántico - Cementos del Caribe.

Berman, M. (1988). Todo lo sólido se desvanece en el aire. La experiencia de la modernidad. Bogotá: Siglo $\mathrm{XXI}$.

Brand, P. (2001). La planeación urbana y las ciencias sociales en Colombia. Revista de Estudios Sociales, (10), 20-30.

Caballero L JE. 2000. Barranquilla y la modernidad. Un ejercicio histórico. Bogotá: PROA - Maestría en Historia y Teoría del Arte y la Arquitectura, Universidad Nacional de Colombia.

Canalización del arroyo de Rebolo. (1950, mayo 10). El Heraldo, p. 8.

Cortés, R. (2007). Del urbanismo a la planeación en Bogotá (1900-1990). Elementos para pensar la trama de un relato. Bitácora Urbano Territorial, 1(11), 160-207.

Crecimiento monstruoso del "Arroyo de Rebolo." (1962, mayo 17). El Heraldo, p. 1, 10.

Currie, L., \& Peñalosa, E. (1952). Plan de fomento para el Atlántico. (L. Currie \& E. Peñalosa, Eds.). Bogotá: Consejo Nacional de Planificación.

Del Castillo, J. C., Urrea, T., Salazar, J., Carrasco, R., Cortés S, R., Espinosa R, L., ... Gonzalez C, M. (2008). Bogotá años 50, el inicio de la metrópoli. Bogotá: Universidad Nacional de Colombia.

Dos millones 171 mil pesos Valor de la ampliación de la carrera 20 de Julio. (1951, enero 16). El Nacional, p. 3.

El "general" invierno y el invierno general. (1955, octubre 29). El Heraldo, p. 8.

El puente de Olaya-Felicidad fue inaugurado. (1962, julio 26). El Heraldo, p. 3.

Este es el proyecto para el arroyo de Rebolo. (1961, febrero 25). El Heraldo, p. 9.

Farias, I., \& Bender, T. (2010). Urban Assemblages. How actor-network theory changes urban studies. London \& New York: Routledge.

Foucault, M. (2006). Seguridad, territorio y población. Buenos Aires: Fondo de Cultura Económica.

García, N. (1989). Culturas híbridas. Estrategias para entrar y salir de la modernidad. México: Gedisa.

Gerlein Comelín, J., \& Gómez Támara, A. (1958, julio 23). Consideraciones sobre la ampliación de Veinte de Julio. El Heraldo, 1, 10.

Goenaga, J. (1959, diciembre 24). La vieja carrera de 20 de julio, es una gran avenida. El Heraldo, p. 3,6. 
Goenaga, J. (1962, agosto 24). El puente de la calle Felicidad. El Heraldo, p. 3.

González Ripoll, R. (1960). Plan de acción para Obras municipales. Boletín de La Cámara de Comercio de Barranquilla, VI(221), p. 5.

Graham, S., \& Marvin, S. (2001). Splintering Urbanism: Network Infrastructures, Technological Mobilities and the Urban Condition. London \& New York: Routledge.

Hall, P. (1996). Ciudades del mañana. Historia del urbanismo del siglo XX. Barcelona: Ediciones del Serbal.

Jensen, O. (2009). Flows of Meaning, Cultures of Movement - Urban Mobility as Meaningful Everyday Life Practice. Mobilities (4)1, 139-158.

La ampliación de 20 de Julio. (1959, diciembre 2). El Heraldo, p. 3.

La ampliación de 20 de Julio fue ordenada de la 44 a la 72. (1955, mayo 11). El Heraldo, p. 5.

Larkin, B. (2013). The Politics and Poetics of Infrastructure. Annual Review of Anthropology, 42(1), 327-343. https://doi.org/10.1146/annurev-anthro-092412-155522.

Latour, B. (2008). Reensamblar lo social. Una introducción a la teoría del actor-red. Buenos Aires: Manantial.

Mazuera, F. (1972). Cuento mi vida. Bogotá: Canal Ramírez - Antares.

McCausland, E (Director). (2010). Ricardo González Ripoll. El arquitecto de la puerta roja. La Esquina del Cine. Retrieved from https://www.youtube.com/watch?v=F4IOqmenjrk

Meisel, A. (1987). ¿Por qué se disipó el dinamismo industrial de Barranquilla? Lecturas de Economía, (23), 55-84.

Mongin, O. (2006). La condición urbana. La ciudad a la hora de la mundialización. Buenos Aires: Paidós.

Nichols, T. (1973). Tres puertos de Colombia. Estudio sobre el desarrollo de Cartagena, Santa Marta y Barranquilla. Bogotá: Biblioteca Banco Popular.

Niño, C., \& Reina, S. (2010). La carrera de la modernidad. Construcción de la Carrera Décima. Bogotá (1945-1960). Bogotá: Alcaldía Mayor de Bogotá, Instituto Distrital de Patrimonio Cultural.

Ospino, P. (2003). "El desarrollo urbano de Barranquilla y su dinámica regional 1777-1993". En Sánchez Bonett, Luis (ed). Barranquilla: lecturas urbanas. Barranquilla: Observatorio del Caribe Colombiano Universidad del Atlántico, pp. 1-44.

Ramírez, J. F. (2011). La Planeación Urbana en Colombia: años sesenta-ochenta. Discursos, consultores y comunidades académicas. Revista de Estudios Sociales, (40), 115-125. Doi:10.7440/res40.2011.11

Salazar, O. I. (2016). Hacer circular y dejar pasar. El ensamblaje de las movilidades urbanas en Bogotá y Barranquilla a mediados del siglo XX (Tesis de doctorado, Universidad Nacional de Colombia - Centro de Estudios Sociales (CES), Bogotá). Descargada de: www.bdigital.unal.edu.co/51660/1/80470134.2016.pdf

Saldarriaga, A. (2000). Bogotá Siglo XX, Arquitectura, Urbanismo y Vida Urbana. Bogotá: Departamento Administrativo de Planeación Distrital.

Salter, M. B. (2013). To Make Move and Let Stop: Mobility and the Assemblage of Circulation. Mobilities, 8(1), 7-19. https://doi.org/10.1080/17450101.2012.747779

Suárez, A. M. (2006). Los juegos de poder detrás de la modernización capitalina: Bogotá, 1946-1948. Anuario Colombiano de Historia Social y de la Cultura, (33), 111-142.

Tecnificación de los impuestos municipales. (1960, agosto 9). Boletín de La Cámara de Comercio de Barranquilla, VI(219), 5-8.

Urry, J. (2007). Mobilities. Cambridge: Polity Press.

Vergara, R. A., \& Vidal, A. (2009). Barrio El Prado. Hito histórico y urbano de Barranquilla. Barranquilla: Ediciones Uninorte. 